\title{
Doses de nitrogênio e molibdênio no rendimento e teor de micronutrientes em alface americana
}

\section{Geraldo M de Resende ${ }^{1}$; Marco Antônio R Alvarenga² ${ }^{2}$ Jony E Yuri ${ }^{3}$ Rovilson José de Souza ${ }^{2}$}

${ }^{1}$ Embrapa Semi-Árido, C. Postal 23, 56302-970 Petrolina-PE; ${ }^{2}$ UFLA-Depto. Agricultura, C. Postal 37, 37200-000 Lavras-MG; ${ }^{3}$ UNINCOR, Av. Castelo Branco 82, 37410-000 Três Corações-MG; gmilanez@cpatsa.embrapa.br; marcoalvarenga@ufla.br; jonyyuri@uol. com.br; rovilson@ufla.br

\section{RESUMO}

O trabalho foi conduzido no município de Três Pontas, Sul de Minas Gerais, de outubro a dezembro de 2002, com o objetivo de avaliar a influência de doses de nitrogênio $(\mathrm{N})$ e molibdênio (Mo) no rendimento e teor de micronutrientes da alface americana. Utilizouse o delineamento de blocos casualizados em arranjo fatorial $4 \mathrm{x}$ 5 , compreendendo quatro doses de $\mathrm{N}\left(0,60,120\right.$ e $\left.180 \mathrm{~kg} \mathrm{ha}^{-1}\right) \mathrm{em}$ cobertura adicionais à dose aplicada pelo produtor de $60 \mathrm{~kg} \mathrm{ha}^{-1} \mathrm{e}$ cinco doses de Mo via foliar $\left(0,0 ; 35,1 ; 70,2 ; 105,3\right.$ e $\left.140,4 \mathrm{~g} \mathrm{ha}^{-1}\right)$ com três repetições. O maior rendimento de massa fresca comercial foi obtido com a dose de $89,1 \mathrm{~kg} \mathrm{ha}^{-1}$ de $\mathrm{N}$ em cobertura e 94,2 $\mathrm{g}$ $\mathrm{ha}^{-1}$ de Mo. Os resultados indicaram incremento nos teores de boro, zinco, ferro e manganês com o aumento das doses de N e Mo. Em relação ao teor de cobre verificou-se efeito significativo da interação $\mathrm{N}$ x Mo, evidenciando para a maior dose de Mo (140,4 $\left.\mathrm{g} \mathrm{ha}^{-1}\right)$ uma redução linear com o aumento das doses de $\mathrm{N}$.

Palavras-chave: Lactuca sativa, rendimento, boro, zinco, cobre, ferro, manganês.

\begin{abstract}
Doses of nitrogen and molybdenum effects on yield and micronutrient content of crisphead lettuce plants

The trial was carried out in Três Pontas, Minas Gerais State, Brazil, from October to December, 2002, to evaluate the influence of doses of nitrogen and molybdenum on yield and macronutrient uptake of crisphead lettuce (Lactuca sativa L.). A randomized complete block design scheme with three replications was used, in which the treatments were a factorial combination of nitrogen rates $(0,60,120$ and $180 \mathrm{~kg} \mathrm{ha}^{-1}$ of $\mathrm{N}$ ) in top dressing in addition to the dose used by farmers $\left(60 \mathrm{~kg} \mathrm{ha}^{-1}\right.$ of $\left.\mathrm{N}\right)$ and five foliar molybdenum rates $(0.0$; $35.1 ; 70.2 ; 105.3$ and $\left.140.4 \mathrm{~g} \mathrm{ha}^{-1}\right)$. The highest yield of commercial fresh mass was obtained using the dose of $89.1 \mathrm{~kg} \mathrm{ha}^{-1}$ of nitrogen in top dressing and $94.2 \mathrm{~g} \mathrm{ha}^{-1}$ of molybdenum. The results indicated an increase in the levels of $\mathrm{Ca}, \mathrm{Zn}, \mathrm{Fe}$ and $\mathrm{Mn}$ as a consequence of the application of higher doses of nitrogen and molybdenum. Significant effect of the interaction $\mathrm{N} x \mathrm{Mo}$ on the $\mathrm{Cu}$ level was verified and it was evidenced, for the highest molybdenum level (140.4 $\left.\mathrm{g} \mathrm{ha}^{-1}\right)$, a linear reduction due to the application of increasing doses of nitrogen.
\end{abstract}

Keywords: Lactuca sativa, yield, boron, zinc, copper, iron, manganese.

(Recebido para publicação em 10 de fevereiro de 2009; aceito em 16 de junho de 2010)

(Received on February 10, 2009; accepted on June 16, 2010)

A alface americana, tipo repolhuda (crisphead lettuce), vem adquirindo importância crescente, principalmente, na região sul de Minas Gerais. O plantio deste tipo de alface visa principalmente atender as redes fast food de alimentação, como MacDonald's (Mota et al., 2003).

A fertilização constitui uma das práticas agrícolas mais caras e de maior retorno econômico, resultando em maiores rendimentos e em produtos mais uniformes e de maior valor comercial (Ricci et al., 1995). A alface é uma planta composta basicamente por folhas e responde à fertilização nitrogenada. A deficiência de $\mathrm{N}$ retarda o crescimento da planta e induz à má formação da cabeça, folhas mais velhas amareladas que desprendem-se com facilidade (Garcia et al., 1982).

O Mo é constituinte de pelo menos cinco enzimas catalisadoras de reações (redutase do nitrato, nitrogenase e oxidase do sulfito) e três são encontradas em plantas (Gupta \& Lipsett, 1981), sendo a sua função mais importante relacionada com o metabolismo do $\mathrm{N}$.

O crescimento da alface, e como conseqüência o acúmulo de nutrientes, é lento até 30 dias após a emergência, aumentando rapidamente após este período. Apesar de absorver quantidades relativamente pequenas de nutrientes devido ao seu ciclo curto (2 a 3 meses), esta cultura pode ser considerada exigente em nutrientes, principalmente na fase final do seu ciclo (Katayama,
1993).

Informações relacionadas à fertilização da alface com os micronutrientes boro, cloro, cobre, ferro, manganês, molibdênio e zinco, são escassas, principalmente em se tratando de alface tipo americana. Entretanto, estes nutrientes são essenciais uma vez que a sua falta impede a planta de completar seu ciclo vital (Malavolta, 2006).

O objetivo deste trabalho foi avaliar os efeitos de doses de $\mathrm{N}$ e de Mo sobre o rendimento e teor de micronutrientes na parte aérea da alface americana cultivada sob condições de verão no sul de Minas Gerais.

\section{MATERIAL E MÉTODOS}


O experimento foi conduzido em propriedade rural, no município de Três Pontas, sul de Minas Gerais, (21 $22^{\prime} 00^{\prime}$ $\mathrm{S} ; 45^{\circ} 30$ '45" $\mathrm{W} ; 870 \mathrm{~m}$ altitude) (IBGE, 2005), em solo classificado como Latossolo Vermelho Distroférrico textura argilosa (Embrapa, 1999). O clima da região é caracterizado por temperatura média anual variando de $15,8^{\circ} \mathrm{C}$ no mês mais frio, a $22,1^{\circ} \mathrm{C}$ no mês mais quente; com precipitação média anual de $1.529,7 \mathrm{~mm}$ e umidade relativa do ar de 76,2\% (Brasil, 1992). A análise química do solo resultou os valores: $\mathrm{K}=70,0 \mathrm{mg} \mathrm{dm}^{-3} ; \mathrm{P}$ (Mehlich) $=78,0$ $\mathrm{mg} \mathrm{dm}{ }^{-3} ; \mathrm{Ca}=4,1 \mathrm{cmol}_{\mathrm{c}} \mathrm{dm}^{-3} ; \mathrm{Mg}=0,8$ $\mathrm{cmol}_{\mathrm{c}} \mathrm{dm}^{-3} ; \mathrm{Al}=0,0 \mathrm{cmol}_{\mathrm{c}} \mathrm{dm}^{-3} ; \mathrm{H}+\mathrm{Al}=$ $2,3 \mathrm{cmol}_{\mathrm{c}} \mathrm{dm}^{-3} ; \mathrm{Zn}=0,8 \mathrm{mg} \mathrm{dm}^{-3} ; \mathrm{Fe}=$ $25,0 \mathrm{mg} \mathrm{dm}^{-3} ; \mathrm{Mn}=14,2 \mathrm{mg} \mathrm{dm}^{-3} ; \mathrm{Cu}=$ $1,0 \mathrm{mg} \mathrm{dm}^{-3} ; \mathrm{B}=0,3 \mathrm{mg} \mathrm{dm}^{-3} ; \mathrm{pH} \mathrm{em}$ $\mathrm{H}_{2} 0=6,0$ e $\mathrm{MO}=24 \mathrm{~g} \mathrm{~kg}^{-1}$.

$\mathrm{O}$ delineamento experimental utilizado foi de blocos ao acaso; os tratamentos distribuídos em fatorial 4 x 5, compreendendo quatro doses de nitrogênio $\left(0,60,120\right.$ e $\left.180 \mathrm{~kg} \mathrm{ha}^{-1}\right) \mathrm{em}$ cobertura adicionais à dose aplicada pelo produtor de $60 \mathrm{~kg}^{\mathrm{de}} \mathrm{N} \mathrm{ha}^{-1}$ e cinco doses de molibdênio aplicadas via foliar $\left(0,0 ; 35,1 ; 70,2 ; 105,3\right.$ e $\left.140,4 \mathrm{~g} \mathrm{ha}^{-1}\right)$, com três repetições. A uréia foi utilizada como fertilizante nitrogenado e o molibdato de sódio como fonte de Mo. A uréia foi aplicada em cobertura aos 10, 20 e 30 dias após o transplante em $40 \%$, $30 \%$ e $30 \%$, respectivamente, da dose total em avaliação. As doses de uréia avaliadas foram previamente diluídas em água pura, aplicando-se $10 \mathrm{~mL}$ da solução, lateralmente a cada planta. O molibdato de sódio foi aplicado aos 21 dias após o transplante por meio de pulverizador costal manual capacidade de 4 L em máxima pressão, gastando-se $300 \mathrm{~L} \mathrm{ha}^{-1}$ de calda.

O preparo do solo foi realizado com aração e gradagem, e os canteiros foram levantados a 0,20 m de altura. As mudas foram feitas em bandejas multicelulares de 288 células cada uma, preenchidas com substrato artificial (Plantmax), sendo o transplante feito aos 25 dias após o semeio, quando as plantas apresentaram dois pares de folhas definitivas, utilizando-se a cultivar Raider.

As parcelas experimentais constituíram-se de canteiros com quatro linhas de 2,1 m de comprimento e espaçamento entre plantas de 0,30 x $0,35 \mathrm{~m}$. As linhas centrais formaram a área útil, retirando-se duas plantas em cada extremidade. Em toda a área foi instalada uma estrutura de proteção, constituída de túneis altos com 2,0 m de altura, cobrindo dois canteiros por túnel, constituído de tubos de ferro galvanizados, coberta com filme plástico transparente de baixa densidade, aditivado com anti-UV, de 100 micras de espessura. Os canteiros foram revestidos com filme plástico preto mulching, de 4 $\mathrm{m}$ de largura e $35 \mu \mathrm{m}$ de espessura.

A fertilização básica de plantio utilizada pelo produtor constou de $30 \mathrm{~kg}$ de $\mathrm{N}+410 \mathrm{~kg}$ de $\mathrm{P}_{2} \mathrm{O}_{5}+120 \mathrm{~kg}$ de $\mathrm{K}_{2} \mathrm{O}$. Após os adubos serem incorporados ao solo, instalou-se em cada canteiro duas linhas de tubo gotejador, com emissores espaçados a cada $30 \mathrm{~cm}$ e com vazão de $1,5 \mathrm{~L} \mathrm{~h}^{-1}$. As adubações em cobertura foram realizadas através de fertirrigações diárias, totalizando $30 \mathrm{~kg}$ de $\mathrm{N}^{-1}$ e 60 $\mathrm{kg}$ de $\mathrm{K}_{2} \mathrm{O} \mathrm{ha}^{-1}$.

A cultura foi mantida no limpo por meio de capinas manuais e o controle fitossanitário foi realizado pelo método padrão do produtor, com pulverizações semanais alternando produtos à base de oxicloreto de cobre, iprodione, procimidone e piretróides, de acordo com a necessidade.

O transplantio das mudas foi realizado 30 dias após a semeadura em 28 de outubro de 2002 e a colheita foi feita em 9 de dezembro de 2002, quando as plantas apresentaram-se completamente desenvolvidas.

Por ocasião da colheita avaliou-se o rendimento de massa fresca comercial ( $\mathrm{g}$ planta $^{-1}$ ) e retiraram-se amostras no terço médio da cabeça comercial de todas as plantas úteis da parcela, obtendo-se uma amostra $( \pm 300 \mathrm{~g})$ por tratamento. Estas foram lavadas em água corrente $\mathrm{e}$ destilada, e secas em estufa com circulação forçada de ar, a $65-70^{\circ} \mathrm{C}$, até peso constante, moídas e acondicionadas em recipientes vedados com tampa de plástico, com as devidas identificações. A análise dos micronutrientes no respectivo material foi realizada em laboratório da UFLA.

O boro foi determinado pelo método colorimétrico da curcumina com digestão por via seca. O zinco, manganês e cobre foram determinados por espectrofotometria de absorção atômica no extrato nitroperclórico. Os demais micronutrientes foram analisados por espectrofotometria de absorção atômica (EAA), de acordo com Malavolta et al. (1997).

Os dados coletados foram submetidos à análise de variância e regressão com base no modelo polinomial a $5 \%$ de probabilidade.

\section{RESULTADOS E DISCUSSÃO}

Houve efeitos significativos das doses de $\mathrm{N}$ e de Mo para massa fresca comercial da parte aérea. As doses de $\mathrm{N}$ foram ajustadas a um modelo quadrático, no qual $89,6 \mathrm{~kg} \mathrm{ha}^{-1} \mathrm{de}$ $\mathrm{N}$ em cobertura resultaram em maior rendimento (Figura 1). Estes resultados são inferiores aos encontrados por Tei et al. (2000), para alface tipo lisa e butterhead, que verificaram maiores produtividades com a dose de $155,0 \mathrm{~kg}$ ha $^{-1}$ de N. Furtado (2001), avaliando doses acima de $148,0 \mathrm{~kg} \mathrm{ha}^{-1}$ de $\mathrm{N}$, não encontrou para massa fresca comercial diferenças significativas.

Em relação às doses de Mo observou-se efeito quadrático com máxima produtividade na dose de 94,2 $\mathrm{g} \mathrm{ha}^{-1}$ (Figura 2). Com o incremento das doses de Mo em função das épocas de aplicação, Yuri et al. (2004) verificaram efeitos quadráticos para massa fresca comercial, tendo as doses de $82,7 \mathrm{~g}$ $\mathrm{ha}^{-1}$ proporcionado o maior rendimento quando aplicado aos 21 dias após o transplantio. Resultados positivos da aplicação de Mo na cultura da alface são relatados por Zito et al. (1994), que observaram aumento médio de 24,1\%, na produção comercial de alface com a aplicação desse micronutriente.

Os teores de boro na parte aérea da alface receberam influência das doses de N e Mo, assim como da interação destes fatores. $\mathrm{O}$ desdobramento da interação Mo x N mostrou efeitos lineares positivos na ausência de Mo e na dose de 70,2 $\mathrm{g} \mathrm{ha}^{-1} \mathrm{de} \mathrm{Mo}$, as quais aumentaram linearmente com o incremento das doses de N (Tabela 1). Na dose de 35,1 $\mathrm{g} \mathrm{ha}^{-1}$ de Mo, ajustou-se um modelo quadrático com teor mínimo de boro $\left(19,8 \mathrm{mg} \mathrm{kg}^{-1}\right)$ 


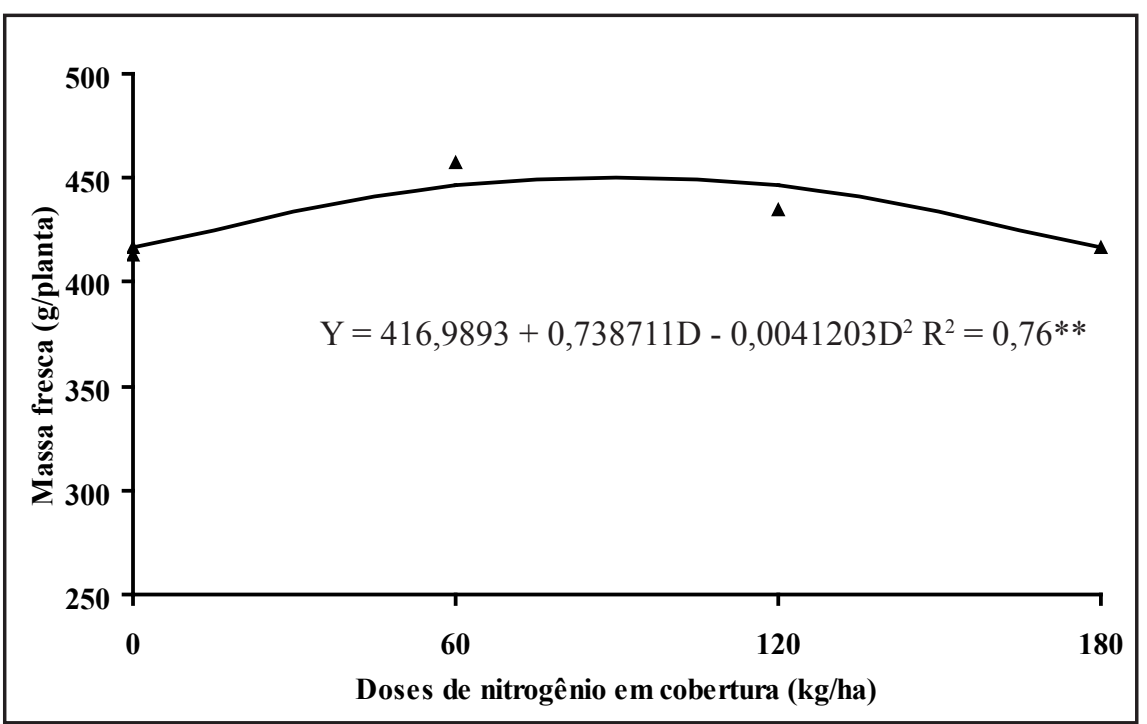

Figura 1. Massa fresca comercial de alface tipo americana em função de doses de nitrogênio (commercial fresh mass of crisphead lettuce in response to nitrogen levels). Três Pontas, Embrapa Semi-Árido, 2002.

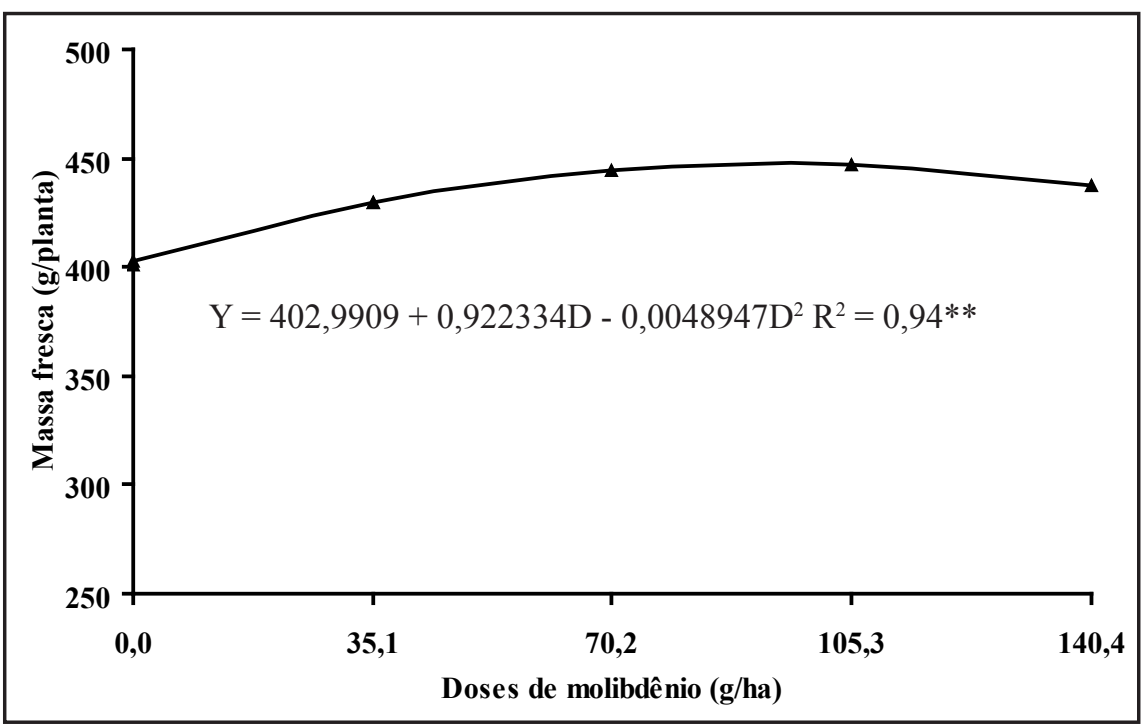

Figura 2. Massa fresca comercial de alface tipo americana em função de doses de molibdênio (commercial fresh mass of crisphead lettuce in response to molybdenum levels). Três Pontas, Embrapa Semi-Árido, 2002.

na dose de $94,2 \mathrm{~g} \mathrm{ha}^{-1}$ de $\mathrm{N}$ em cobertura. Para a dose de 105,3 $\mathrm{g} \mathrm{ha}^{-1}$ de Mo verificou-se efeito quadrático com maior teor de boro $\left(16,8 \mathrm{mg} \mathrm{kg}^{-1}\right)$ na dose de 106,6 $\mathrm{kg} \mathrm{ha}^{-1}$ de N. Estes resultados estão coerentes com os obtidos por Resende et al. (1997) que constataram aumento linear no teor de boro na parte aérea do milho, com o incremento das doses de $\mathrm{N}$, atribuindo este fato, ao papel do $\mathrm{N}$ no desenvolvimento da planta. Furtado (2001) também encontrou tendência de maior teor de boro com o incremento das doses de $\mathrm{N}$ em alface americana.

Foram constatados efeitos significativos das doses de $\mathrm{N}$ e Mo e da interação destes fatores no teor de zinco na parte aérea da alface. Desdobrando-se a interação Mo x N verificou-se um efeito linear positivo para a dose de $35,1 \mathrm{~g}$ de Mo ha-1 ${ }^{-1}$ a qual aumentou com o incremento das doses de $\mathrm{N}$ (Tabela 1). Na ausência de Mo, a dose de 74,6 kg de $\mathrm{N} \mathrm{ha}^{-1}$ em cobertura promoveu o maior teor de $\mathrm{Zn}$. Os maiores teores de $\mathrm{Zn}$ foram obtidos para as interações entre as doses de 70,2, 105,3 e 140,4 $\mathrm{g} \mathrm{ha}^{-1} \mathrm{de}$ Mo e 102,1, 113,3 e 86,3 $\mathrm{kg} \mathrm{ha}^{-1}$ de N. Também foram observados efeitos positivos da fertilização nitrogenada sobre o teor de zinco na parte aérea de batata (Soltanpour, 1969) e milho (Resende et al., 1997), assim como em Ligustrum vulgare (Stratton et al, 2001). Furtado (2001) porém não observou diferença significativa no teor de zinco em função da aplicação de doses de $\mathrm{N}$ em alface americana.

Resultados similares foram observados em relação ao teor de cobre na parte aérea da alface (Tabela 1). Na análise da interação Mo x N foram ajustados modelos quadráticos, evidenciando para a maior dose de Mo $\left(140,4 \mathrm{~g} \mathrm{ha}^{-1}\right)$ um efeito linear depressivo com o aumento das doses de N. Na ausência da fertilização com Mo, nas doses de 70,2 e 105,3 $\mathrm{g} \mathrm{ha}^{-1}$, ajustaram-se modelos quadráticos com pontos de máximo teor de cobre nas doses de 62,2, 89,8 e 92,2 $\mathrm{kg} \mathrm{ha}^{-1} \mathrm{de}$ $\mathrm{N}$ em cobertura. Para a dose de $35,1 \mathrm{~g}$ ha $^{-1}$ de Mo houve ajuste quadrático com ponto de mínimo teor de cobre na dose de $113,3 \mathrm{~g} \mathrm{de}$ ha $^{-1}$. Furtado (2001) não observou aumento no teor de cobre em alface americana submetida a aplicações de doses crescentes de N, bem como Alvarenga et al. (2000) quando aplicaram $\mathrm{N}$ e cálcio. No entanto, Resende $e t$ al. (1997) constataram aumento linear no teor de cobre na parte aérea do milho com as doses de N. Stratton et al. (2001) observaram maior teor de cobre na parte aérea de ligustrum (privet) em função da aplicação de N. Segundo Santos (1991), há um efeito de inibição não competitiva entre os íons $\mathrm{MoO}_{4}^{2-}$ e $\mathrm{Cu}^{2+}$, isto é, o inibidor se combina com o sítio não ativo do carregador.

Observaram-se efeitos significativos das doses de N, Mo e da interação $\mathrm{N} x$ Mo sobre o teor de ferro na parte aérea da alface. $\mathrm{O}$ desdobramento da interação entre doses de $\mathrm{N}$ e de Mo ajustou modelos quadráticos para todas as doses (Tabela 1). Na ausência de Mo obteve-se a dose de 133,9 kg de $\mathrm{N}^{-1}$ como a que proporcionou maior teor de ferro. Para as doses de 35,1; 70,2; 105,3 e 140,4 $\mathrm{g} \mathrm{ha}^{-1}$ de Mo estimou-se as doses 119,6; 95,6; 99,1 e 95,8 $\mathrm{kg} \mathrm{ha}^{-1}$ de $\mathrm{N}$, respectivamente, como as que resultaram em maior teor de Fe. Furtado (2001) observou tendência de maior teor de ferro com o incremento das doses de $\mathrm{N}$ em alface americana. Resende et al. (1997) obtiveram maior teor de Fe na parte aérea de plantas de milho com fertilização nitrogenada. 
Tabela 1. Teores de micronutrientes na parte aérea de alface americana em função de doses de nitrogênio e de molibdênio $\left(\mathrm{kg} \mathrm{ha}^{-1}\right)$ (micronutrients content of crisphead lettuce aboveground part in response to nitrogen and molybdenum levels $\left.\left(\mathrm{kg} \mathrm{ha}^{-1}\right)\right)$. Três Pontas, Embrapa Semi-Árido, 2002.

\begin{tabular}{|c|c|c|}
\hline $\begin{array}{c}\text { Micro- } \\
\text { nutrientes } \\
\left(\mathrm{mg} \mathrm{kg}^{-1}\right)\end{array}$ & Equações de regressão & $\mathrm{R}^{2}$ \\
\hline \multirow{4}{*}{ Boro } & $\mathrm{Y}(0,0)=16,0953+0,007755^{*} \mathrm{D}$ & 0,98 \\
\hline & $\mathrm{Y}(35,1)=\mathrm{Y}=18,1116-0,036444 \mathrm{D}+0,0001935^{* *} \mathrm{D}^{2}$ & 0,98 \\
\hline & $\mathrm{Y}(70,2)=15,1883+0,007666 * \mathrm{D}$ & 0,96 \\
\hline & $\mathrm{Y}(105,3)=11,7701+0,095086 \mathrm{D}-0,0004461 * \mathrm{D}^{2}$ & 0,86 \\
\hline \multirow{5}{*}{ Zinco } & $\mathrm{Y}(0,0)=41,1930+0,129411 \mathrm{D}-0,0008675 * * \mathrm{D}^{2}$ & 0,88 \\
\hline & $\mathrm{Y}(35,1)=41,3926+0,034655^{* *} \mathrm{D}$ & 0,99 \\
\hline & $\mathrm{Y}(70,2)=40,6691+0,049875 \mathrm{D}-0,0002442 * * \mathrm{D}^{2}$ & 0,96 \\
\hline & $Y(105,3)=39,2673+0,037122 \mathrm{D}-0,0001638 * \mathrm{D}^{2}$ & 0,77 \\
\hline & $\mathrm{Y}(140,4)=36,9843+0,051516 \mathrm{D}-0,0002986 * * \mathrm{D}^{2}$ & 0,92 \\
\hline \multirow{5}{*}{ Cobre } & $\mathrm{Y}(0,0)=7,0993+0,018988 \mathrm{D}-0,0001527 * * \mathrm{D}^{2}$ & 0,91 \\
\hline & $\mathrm{Y}(35,1)=7,4318-0,013163 \mathrm{D}+0,0000581^{* *} \mathrm{D}^{2}$ & 0,99 \\
\hline & $\mathrm{Y}(70,2)=6,9896+0,012050 \mathrm{D}-0,0000671 * * \mathrm{D}^{2}$ & 0,99 \\
\hline & $\mathrm{Y}(105,3)=6,4410+0,009127 \mathrm{D}-0,0000495^{*} \mathrm{D}^{2}$ & 0,93 \\
\hline & $\mathrm{Y}(140,4)=6,1466-0,003388 * * \mathrm{D}$ & 0,96 \\
\hline \multirow{5}{*}{ Ferro } & $\mathrm{Y}(0,0)=91,2893+0,191239 \mathrm{D}-0,0007143 * * \mathrm{D}^{2}$ & 0,88 \\
\hline & $\mathrm{Y}(35,1)=94,5004+0,191064 \mathrm{D}-0,0007988^{* *} \mathrm{D}^{2}$ & 0,72 \\
\hline & $\mathrm{Y}(70,2)=95,1256+0,083816 \mathrm{D}-0,0004384 * \mathrm{D}^{2}$ & 0,95 \\
\hline & $\mathrm{Y}(105,3)=95,1406+0,091150 \mathrm{D}-0,0004597 * \mathrm{D}^{2}$ & 0,99 \\
\hline & $\mathrm{Y}(140,4)=94,4838+0,148952 \mathrm{D}-0,0007775 * * \mathrm{D}^{2}$ & 0,84 \\
\hline \multirow{5}{*}{ Manganês } & $\mathrm{Y}(0,0)=23,6739+0,123483 \mathrm{D}-0,0004588^{* *} \mathrm{D}^{2}$ & 0,97 \\
\hline & $\mathrm{Y}(35,1)=21,6896+0,051188 * * \mathrm{D}$ & 0,89 \\
\hline & $Y(70,2)=22,6886+0,048366 * * D$ & 0,98 \\
\hline & $Y(105,3)=22,4356+0,022900 * * D$ & 0,95 \\
\hline & $\mathrm{Y}(140,4)=19,8436+0,067283 *{ }^{*} \mathrm{D}$ & 0,82 \\
\hline
\end{tabular}

**Significativo pelo teste de $\mathrm{F}, \mathrm{p}<0,01$ (significant through $\mathrm{F}$ test, $\mathrm{p}<0,01$ ); * Significativo pelo teste de $\mathrm{F}, \mathrm{p}<0,05$ (significant through $\mathrm{F}$ test, $\mathrm{p}<0,05$ ).

A análise do teor de manganês na parte aérea da alface mostrou efeitos significativos das doses de $\mathrm{N}$ e Mo e da interação entre esses nutrientes (Tabela 1). Pelo desdobramento da interação verificou-se na ausência de Mo, um ajuste quadrático, no qual a dose de 134,6 $\mathrm{kg} \mathrm{ha}^{-1}$ de $\mathrm{N}$ em cobertura resultou em maior teor de manganês. Para as demais doses de Mo ajustou-se modelos lineares positivos, ou seja, com o incremento das doses de $\mathrm{N}$ houve incremento no teor de manganês. Furtado (2001) observou uma tendência de aumento do teor de manganês na parte aérea de alface americana com o aumento das doses de $\mathrm{N}$,
ALVARENGA MAR; SILVA EC; SOUZA RJ; CARVALHO JG. 2000. Efeito de doses de nitrogênio aplicadas no solo e níveis de cálcio aplicados via foliar sobre o teor e o acúmulo de micronutrientes em alface americana. Ciência e Agrotecnologia 24: 905-916.

BRASIL. Ministério da Agricultura e Reforma Agrária. 1992. Normas climatológicas - 19611990. Brasília: MARA. 84 p.

EMPRESA BRASILEIRA DE PESQUISA AGROPECUÁRIA. 1999. Sistema brasileiro de classificação de solos. Brasília: Embrapa Produtividade de Informações (SPI). 412p.

FURTADO SC. 2001. Nitrogênio e fósforo na produtividade e nutrição mineral de alface americana cultivada em sucessão ao feijão após o pousio da área. Lavras: UFLA. 78p. (Tese mestrado).

GARCIA LCL; HAAG HP; DIEHL NETO V. 1982. Nutrição mineral de hortaliças XXXVIII: Deficiências de macronutrientes em alface (Lactuca sativa L.), cv. Brasil 48 e Clause's Aurélia. Anais da Escola Superior de Agricultura Luiz de Queiroz 39: 349-372.

GUPTA UC; LIPSETT J. Molybdenum in soils, plants, and animals. 1981. Advances in Agronomy 34: 73-115.

IBGE. 2005. Organização do território - vilas e cidades. Disponível em: http:// www.Ibge.gov. br. Acessado em 19 de fevereiro de 2005.

KATAYAMA M. 1993. Nutrição e adubação de alface, chicória e almeirão. In: SIMPÓSIO SOBRE NUTRIÇÃO E ADUBAÇÃO DE HORTALIÇAS, Jaboticabal. Anais... Piracicaba: POTAFOS, cap. 4, p. 141-148.

MALAVOLTA E. 2006. Manual de nutrição mineral de plantas. São Paulo: Editora Agronômica Ceres. 638p.

MALAVOLTA E; VITTI GC; OLIVEIRA SA. 1997. Avaliação do estado nutricional das plantas: princípios e aplicações. 2. ed. Piracicaba: Associação Brasileira para a Pesquisa da Potassa e do Fosfato. 319p.

MOTA JH; YURI JE; FREITAS SAC; RODRIGUES JUNIOR JC; RESENDE GM; SOUZA RJ. 2003. Avaliação de cultivares de alface americana durante o verão em Santana da Vargem, MG. Horticultura Brasileira 21: 234-237.

RESENDE GM; SILVA GL; PAIVA LE; DIAS PF; CARVALHO JG. 1997. Resposta do milho (Zea mays L.) a doses de nitrogênio e potássio em solo da região de Lavras-MG. III. Micronutrientes na parte aérea. Ciência e Agrotecnologia 21: 71-76.

RICCI MSF; CASALI VWD; CARDOSO AA; RUIZ HA. 1995. Teores de nutrientes em duas cultivares de alface adubadas com composto orgânico. Pesquisa Agropecuária Brasileira 30: 1035-1039.

SANTOS OS. 1991. Molibdênio. In: FERREIRA ME; CRUZ MCP. (eds). Micronutrientes na Agricultura. Piracicaba: POTAFQS/CNPq. p. 191-217.

SOLTANPOUR PN. 1969. Effect of nitrogen, phosphorus and zinc placement on yield and composition of potatoes. Agronomy Journal 61: 288-289.

STRATTON ML; GOOD GL; BARKER AV. 
2001. The effects of nitrogen source and concentration on the growth and mineral composition of privet. Journal of Plant Nutrition 124: 1745-1772.

TEI F; BENINCASA P; GUIDUCCI M. 2000. Effect of nitrogen availability on growth and nitrogen uptake in lettuce. Acta Horticulturae
533: 385-392.

YURI JE. 2004. Produção, nutrição e conservação pós-colheita da alface tipo americana, cv. Raider, no verão e no inverno, em função da aplicação de nitrogênio e potássio em cobertura. Lavras: UFLA. 139p. (Tese doutorado).
ZITO RK; FRONZA V; MARTINEZ HEP; PEREIRA PRG; FONTES PCR. 1994. Fontes de nutrientes, relações nitrato:amônio e molibdênio, em alface (Lactuca sativa L.) produzida em meio hidropônico. Revista Ceres 41: 419-430. 